\title{
EFFECTS OF PENTOBARBITAL ANESTHESIA ON THE PLASMA CATECHOLAMINES AND RENIN ACTIVITY AS REFLECTED IN THE HEMODYNAMIC CHANGES IN DOGS
}

\author{
Makie HIGUCHI and Takeo ASAKAWA \\ Department of Pharmacology, Saga Medical School. Nabeshima. Saga 840-01. Japan
}

Accepted October 12, 1982

\begin{abstract}
Correlation between hemodynamic changes and plasma catecholamines or renin activity were studied in dogs anesthetized with pentobarbital, $30 \mathrm{mg} / \mathrm{kg}$, i.v. Pentobarbital increased heart rate in all cases regardless of changes in plasma catecholamines, and the increase was not depressed fully by propranolol. Blood pressure (BP) showed a transient decline just after pentobarbital injection and then elevated gradually to one of three levels $30-60 \mathrm{~min}$ after anesthesia. In low $(62 \pm 4 \mathrm{mmHg}$. Mean \pm S.E.) and very high BP $(169 \pm 7 \mathrm{mmHg})$, plasma norepinephrine (NE) showed low $(305 \pm 55 \rightarrow 89 \pm 22$ $\mathrm{pg} / \mathrm{ml})$ and high levels $(296 \pm 54 \rightarrow 372 \pm 106 \mathrm{pg} / \mathrm{ml})$, respectively, which probably reflect the neurosympathetic activities. In moderately high $\mathrm{BP}(112 \pm 4 \mathrm{mmHg})$. however. the hypertension did not necessarily reflect changes in plasma NE. When the level before anesthesia was 108-164 pg/ml, plasma NE increased, whereas it decreased when

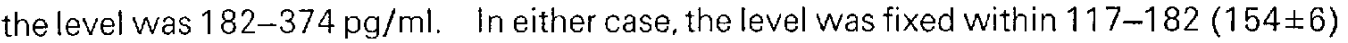
$\mathrm{pg} / \mathrm{ml}$. Plasma renin activity increased after anesthesia and maintained for at least $2 \mathrm{hr}$. However, the increase were observed regardless of the BP level, and moderately high BP was not depressed by an angiotensin 11 antagonist. Participation of angiotensin in these sustained BP cases seems unlikely. Plasma epinephrine reflecting adrenalmedullary activity was decreased markedly in all cases, and the low level lasted for at least $2 \mathrm{hr}$. Changes in plasma dopamine are related to those in plasma NE, and the origin seems to be the same.
\end{abstract}

General anesthesia can alter the responses of the cardiovascular system to a variety of physiological and pharmacological stimuli (1). Sodium pentobarbital (PB) is the most commonly used general anesthetic in experimental pharmacology. When a dog is anesthetized with PB, $30 \mathrm{mg} / \mathrm{kg}$ i.v., the hemodynamics usually exhibits a behavior which seems to result from an enhanced sympathetic nerve tone: persistent hypertension following a transient hypotension and significant increase in heart rate. On the other hand, the depressive effects of $P B$ on transmitter release in autonomic nervous systems have been reported in isolated preparations. Thus, an anesthetic concen- tration of $\mathrm{PB}$ inhibits the catecholamine release induced by activation of the nicotinic receptors on the sympathetic nervous systems $(2,3)$ as well as causing a direct inhibition of the postganglionic release of acetylcholine (ACh) (4). In in situ studies, also. PB anesthesia reduces the catecholamine secretion of the adrenal medulla in dogs (5) and decreases plasma catecholamines $15 \mathrm{~min}$ after $P B$ injection in rats (6). Besides, concentrations of PB appropriate to induce surgical anesthesia can exert direct depressant effects on vascular smooth muscle (7) and on the cardiac conduction system $(8,9)$.

It is conceivable that in situ, various reflex systems overcome the direct inhibiting 
effects. However, little work has yet been done as to how much correlation there is between the effects of PB anesthesia on hemodynamics and those on plasma catecholamines, which may indicate directional changes in sympathoadrenal activity (10). The present investigation was done in the hope of clarifying the above correlation. specifically with passage of time, and the under-anesthesia responses to exogenous norepinephrine (NE) in relation to plasma $N E$ concentration. In view of the fact that an increase in plasma renin activity by $P B$ anesthesia (11-13) and angiotensin 11 mediated facilitation of adrenergic neurotransmission (14) have been observed, to what extent angiotensin II participates in post-anesthetic hypertension has also been examined by use of an angiotensin II antagonist.

\section{Materials and Methods}

General: Experiments were performed on 22 mongrel dogs of either sex weighing 8-14 $\mathrm{kg}$. The dogs were given PB injection. $30 \mathrm{mg} / \mathrm{kg}$. into the superficial vein of the forearm and reached anesthetic states without corneal reflexes. They were divided into 4 groups according to the systemic blood pressure levels 30-60 min after anesthesia. In Group I (4 dogs), Group II (14 dogs) and Group III (4 dogs), the blood pressure

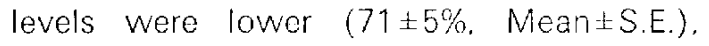

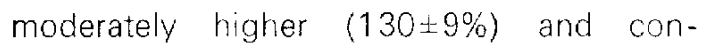
siderably higher $(197 \pm 6 \%)$ than those before anesthesia (100\% as control). Group IV (4 dogs) were given propranolol, $10 \mathrm{mg} / \mathrm{kg} /$ day p.o., for 2 weeks, and they were anesthetized about $14 \mathrm{hr}$ after the last administration of propranolol. Statistical analysis was done using the Student's $t$-test.

Measurements of heart rate and systemic blood pressure: The dogs were moved from the breeding room to the experimental room and left undisturbed for more than $1 \mathrm{hr}$.
They were allowed to rest for $30 \mathrm{~min}$ in a sitting state or lying state in some cases until the heart rate and blood pressure stabilized; Measurements were then taken. Blood samples were then collected, and the PB injections were done in the same states. The heart rate in consciousness was counted from the electrocardiogram lead II (Nihon Kohden, Model AB-620G) and after anesthesia with a cardiotachometer (Nihon Kohden, Model AT-600 G). Systemic blood pressure in consciousness was measured every min from the forearm by the oscillometric method (Biochem, SENSOMAT PB) in 6 cases. In 5 cases, it was obtained from a side tube of a T-type silicone catheter implanted in the right carotid artery at least 3 days before the experiments using an electronic manometer (Nihon Kohden. Model MPU-0.5). In 15 cases, blood pressure was measured only after anesthesia from a cannula inserted into the femoral artery. These measurements were continuously recorded (Nihon Kohden, Model RM 6000).

Measurements of plasma catecholamines and renin activity: Blood samples of $1 \mathrm{ml}$ and $3 \mathrm{ml}$ for measuring plasma catecholamine concentrations and plasma renin activity. respectively, were collected into pre-chilled tubes containing EGTA Na from the superficial vein of the forearm just before and 30-60 min after anesthesia. In 7 cases, blood samples were collected 30,60 and $120 \mathrm{~min}$ after the injection of PB. The blood samples were centrifuged at $3.000 \mathrm{pm}$ for $15 \mathrm{~min}$ at $4^{\circ} \mathrm{C}$. and the supernatants were stored below $-20^{\circ} \mathrm{C}$ until they were analyzed. Plasma catecholamines (NE, epinephrine: Epi, dopamine: DA) were determined by radioenzymatic assay using a CAT-A-KIT (Upjohn). Plasma renin activity was determined by radioimmunoassay using a RENIN. RIAKIT (Dainabot).

Hemodynamic responsiveness to exogenous NE, propranolol and angiotensin II 
antagonist: Under anesthesia, agents were injected into the saphenous vein. In 15 cases, the effects of exogenous NE, 0.03-3 $\mu \mathrm{g} / \mathrm{kg}$, on blood pressure were measured. In 5 cases, the effects of rapid injection (bolus) of 1 -sarcosine, 8 -isoleucine angiotensin II (1-Sar-8-lle-angiotensin II, Daiichi Seiyaku Co., Tokyo), $20 \mu \mathrm{g} / \mathrm{kg}$, and a further $40 \mu \mathrm{g} / \mathrm{kg}$ bolus at $12 \mathrm{~min}$ after the first injection were measured. In 3 cases, the effects of propranolol, $0.03-10 \mathrm{mg} / \mathrm{kg}$, on heart rate were measured.

\section{Results}

Changes in hemodynamics, plasma catecholamines and plasma renin activity: Systemic blood pressure showed a transient decline just after the injection of PB and then elevated gradually to three distinct levels 30-60 min after anesthesia (Table 1). Figure 1 gives representative hemodynamic changes with the passage of time ( $2 \mathrm{hr}$ after anesthesia) obtained in low blood pressure (Group 1) and moderately high blood pressure (Group II) groups. Plasma catecholamine concentrations and plasma renin activity under these conditions are shown in Fig. 2. The measurements 30-60 min after anesthesia are summarized in Table 1.

In Group I, the decreased blood pressure slowly recovered nearly to the preanesthetic levels 60-90 min after anesthesia and maintained the levels for $1 \mathrm{hr}$. In most cases (Group II), at $30 \mathrm{~min}$ after anesthesia, the blood pressure was already over the preanesthetic levels, and moderately high levels (100-142 $\mathrm{mmHg}$ ) lasted thereafter. Heart rate showed a transient, though marked, increase just after anesthesia and immediately declined; but in all cases, it remained on the level significantly higher than the preanesthetic. Percentile changes 30-60 min after anesthesia in Group I, II and III were

Table 1. Effects of pentobarbital anesthesia on blood pressure, heart rate, plasma catecholamines and plasma renin activity in dogs

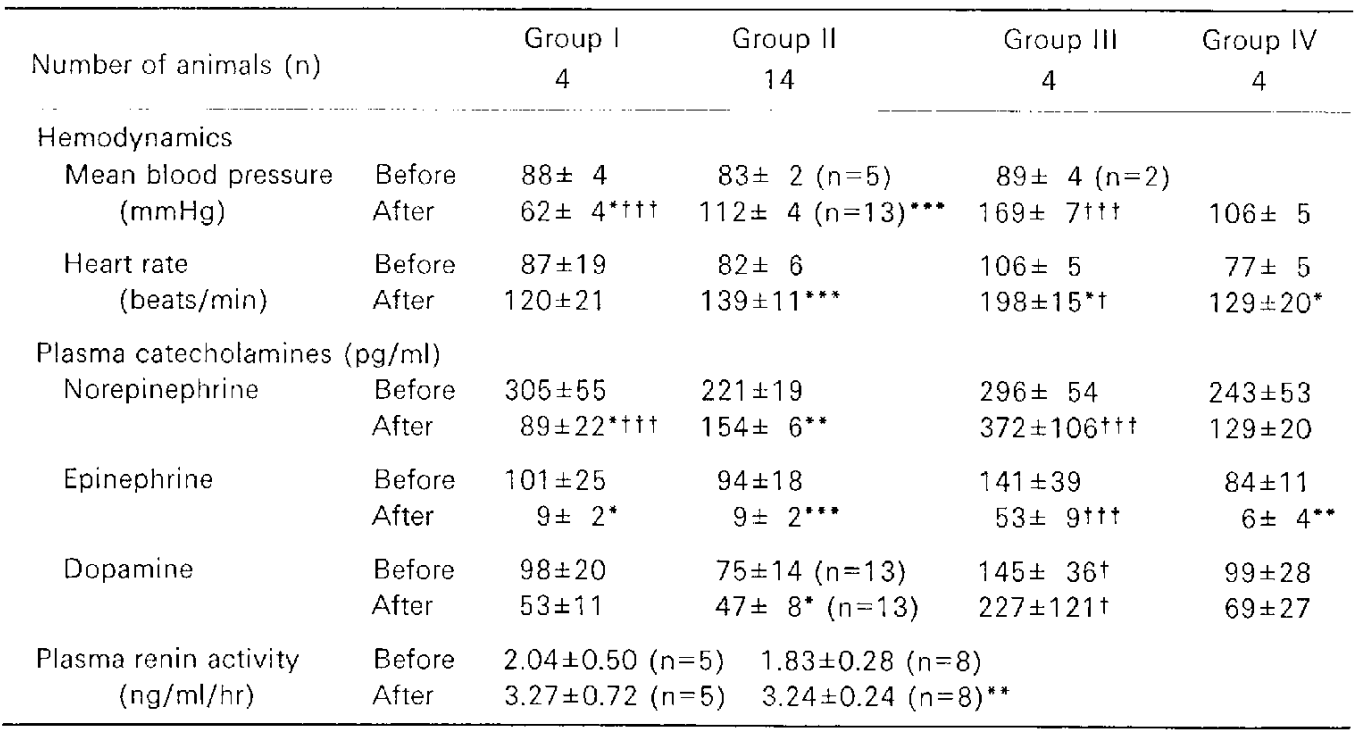

Mean \pm S.E. * ${ }^{* *},{ }^{* *}$ : Significantly different from the control values obtained before anesthesia at $P<0.05,0.01$ and 0.001 , respectively, $\dagger, \dagger^{+}:$Significantly different from Group $\|$at $P<0.05$ and 0.001 , respectively. Groups were classified into low (Group I). moderately high (Group II) and very high blood pressure (Group $1: 1$ ) according to the levels $30-60 \mathrm{~min}$ after injection of sodium pentobarbital ( $30 \mathrm{mg} / \mathrm{kg}$ i.v.). Group IV was anesthetized after a two week-treatment of propranolol (10 $\mathrm{mg} / \mathrm{kg} /$ day p.o.). 
Group I

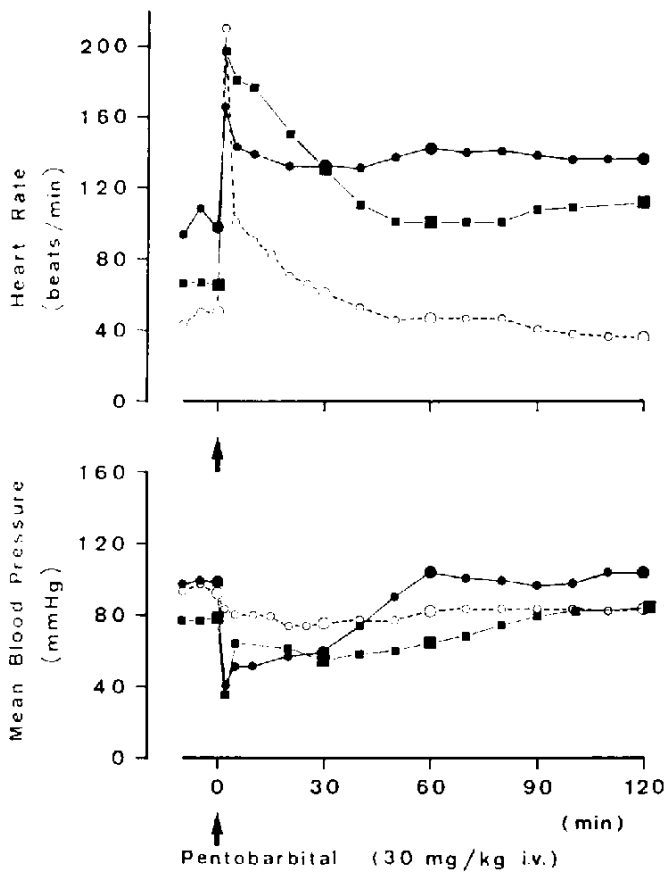

Group II

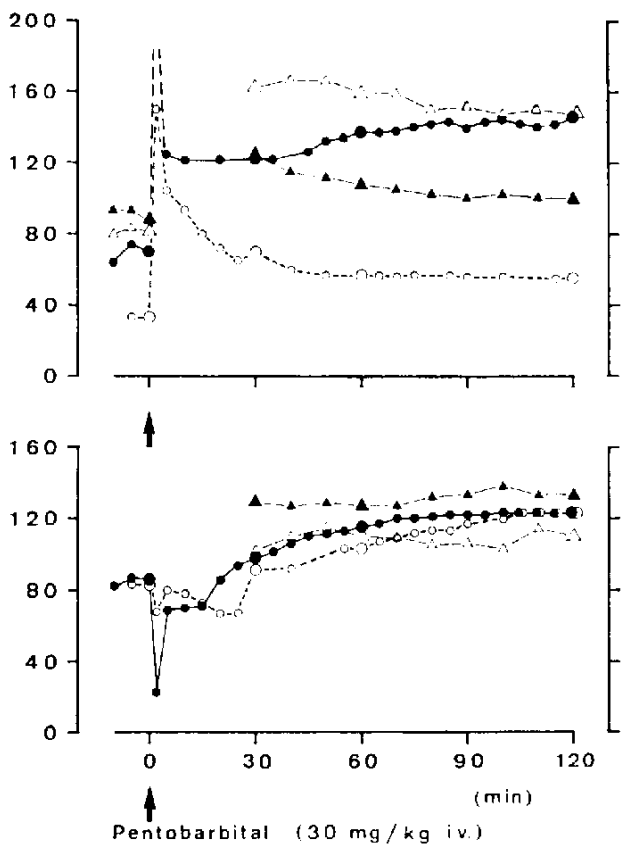

Fig. 1. Changes in heart rate and systemic blood pressure for $2 \mathrm{hr}$ after pentobarbital anesthesia in some examples classified into low (Group I) and moderately high blood pressure (Group II). Each different symbol represents a different sample.

$143 \pm 19 \%, 177 \pm 14 \%$ and $189 \pm 20 \%$, respectively. These elevated heart rates were depressed by $17 \pm 3 \% \quad(n=3)$ following an injection of propranolol, $0.3 \mathrm{mg} / \mathrm{kg}$ i.v.

In Group II, plasma NE after anesthesia showed a slight decrease or increase depending on the level before anesthesia, stayed on almost the same level during the 30 to $60 \mathrm{~min}$ period after anesthesia and tended to reach a higher level $120 \mathrm{~min}$ after anesthesia. When the preanesthetic level was within 108-164 $\mathrm{pg} / \mathrm{ml}(\mathrm{n}=3)$, plasma NE showed an increase, whereas a preanesthetic level within $182-374 \mathrm{pg} / \mathrm{ml}(\mathrm{n}=11)$ exhibited a decrease. In either case, the level was within the range of $117-182 \mathrm{pg} / \mathrm{ml} 30-60$ min after anesthesia. Plasma NE in Group 1 decreased significantly $30 \mathrm{~min}$ after anesthesia, and the levels were lower than those of Group II. Sixty min after anesthesia. NE levels increased slightly, but they were still lower than the preanesthetic levels; these levels were maintained thereafter. On the other hand, in very high blood pressure after anesthesia (Group III), the plasma NE levels that showed increases in 3 out of 4 cases independent of the preanesthetic level were significantly higher than in Group II. Plasma Epi showed a significant decrease in all cases regardless of the changes in blood pressure after anesthesia, but the decrease in Group 111 was smaller than in the other groups. The decreased levels lasted for at least $2 \mathrm{hr}$. Plasma DA tended to decrease in Groups I and II and increase in Group III. There was a significant linear relationship between the percentile changes in plasma NE and DA after PB anesthesia (Fig. 3).

$P B$ anesthesia induced significant increases in plasma renin activity regardless of the 

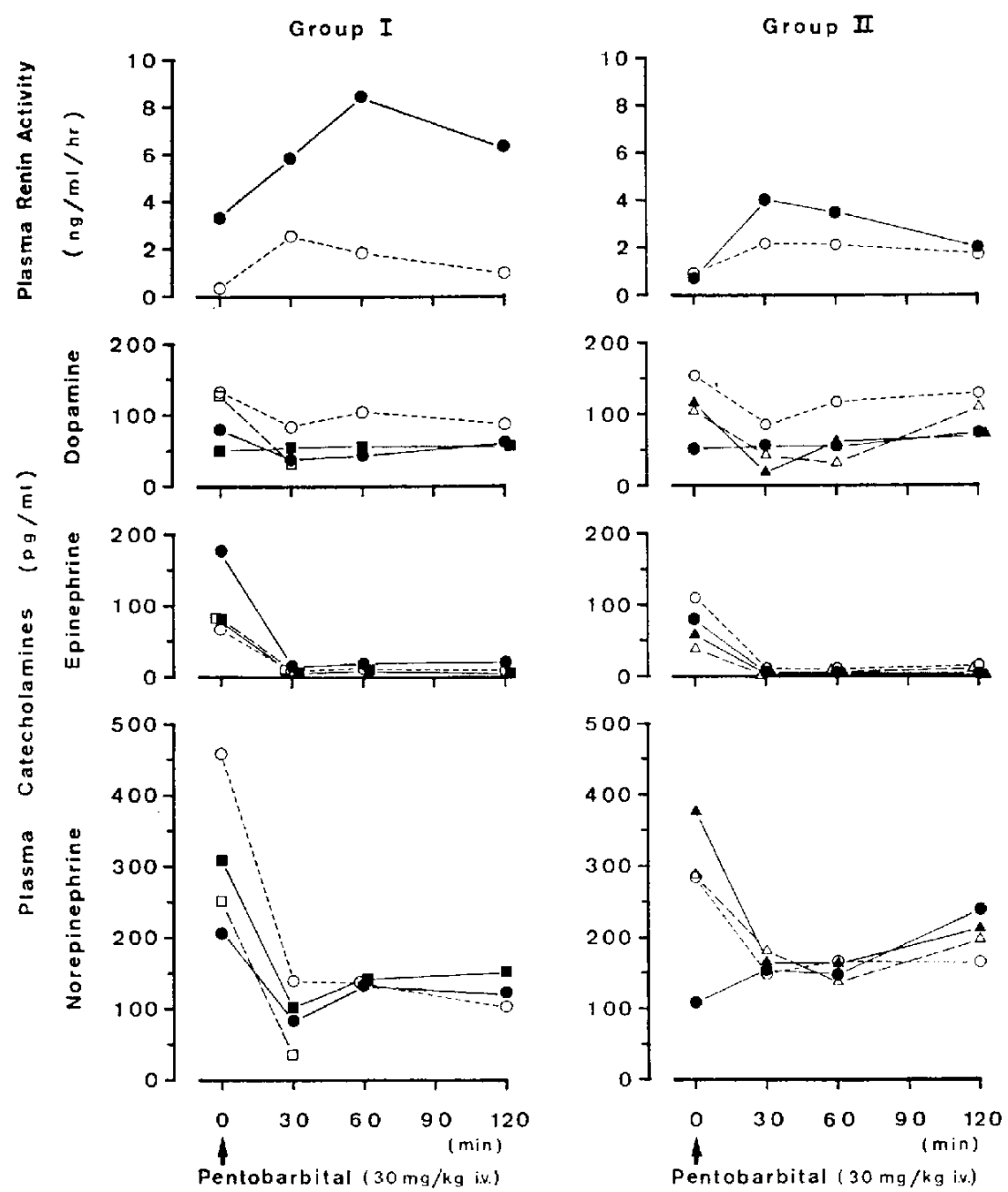

Fig. 2. Changes in plasma catecholamine concentrations and plasma renin activity for 2 hr after pentobarbital anesthesia in some examples classified into low (Group I) and moderately high blood pressure (Group II). Same symbol corresponds to the same sample as described in Fig. 1.

blood pressure levels after anesthesia in Groups I and II. The maximal levels were found within $60 \mathrm{~min}$. Then a tendency to return towards the preanesthetic levels was observed, but the levels remained higher for at least $2 \mathrm{hr}$.

In propranolol-pretreated cases (Group IV). heart rate also showed a significant increase (167 $\pm 17 \%)$, and the changes in plasma catecholamines were almost the same as in Group II.

NE sensitivity: Responsiveness to exoge- nous NE were determined by measuring the degree of increase in blood pressure in Groups II, III and IV (Fig. 4). The levels of responsiveness to NE were similar between Groups II and IV, and it was significantly less pronounced in Group III than in the other two groups.

Effects of angiotensin II antagonist: Effects of 1-Sar-8-lle-angiotensin II on moderately high blood pressure under $\mathrm{PB}$ anesthesia are shown in Fig. 5. The injection of the agent, $20 \mu \mathrm{g} / \mathrm{kg}$ i.v.. induced a 
transient increase of blood pressure, and the increase was more prominent in diastolic than in systolic pressure. Seven min after the

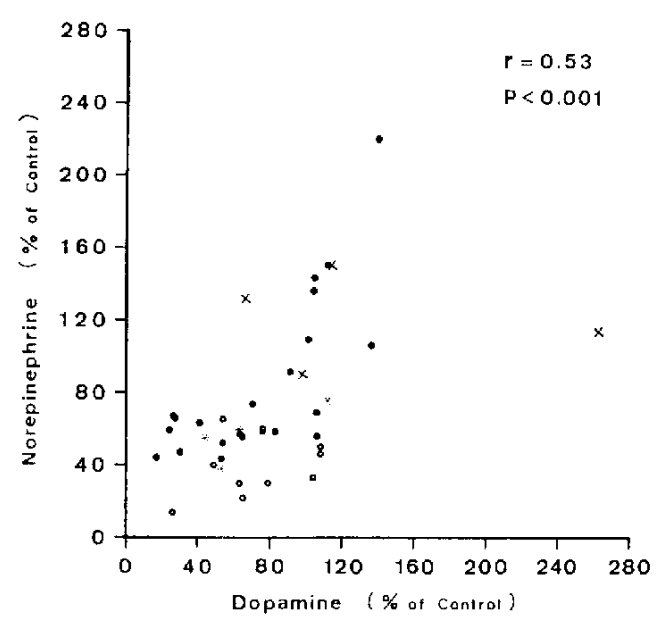

Fig. 3. Relationship between the percentile changes in plasma norepinephrine and dopamine after pentobarbital anesthesia. (O), (O), (x) and $\left({ }^{*}\right)$ represent the values obtained in Group I, II, III and IV, respectively. injection, it had returned to premedication levels, and thereafter, no decrease was

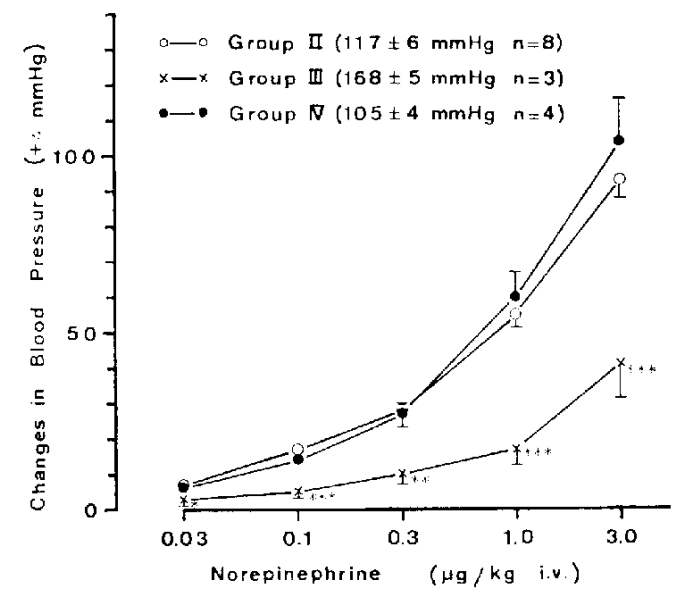

Fig. 4. Responses of blood pressure to exogenous norepinephrine in dogs anesthetized with pentobarbital. Vertical lines are standard errors of the means. The values in parentheses represent the premedication-levels of blood pressure. ".**,"*** Significantly different from the Group $\|$ at $P<0.05$. 0.01 and 0.001 , respectively.

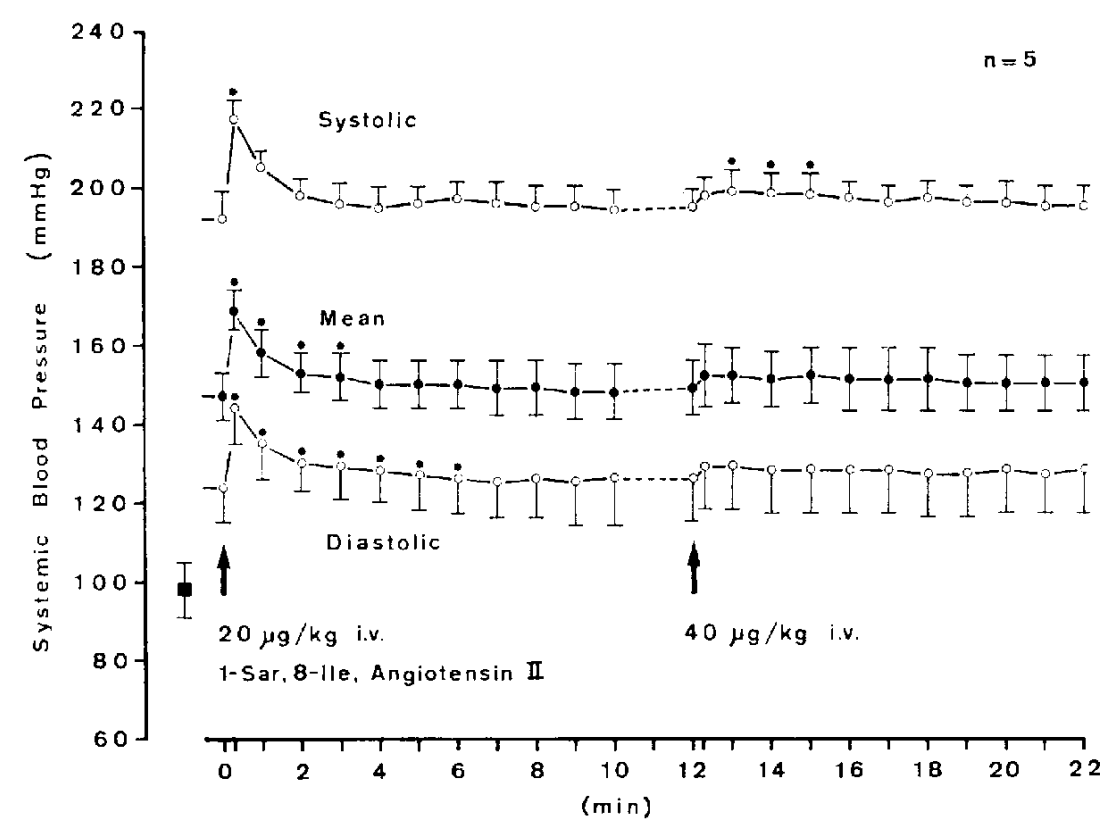

Fig. 5. Effects of an angiotensin 11 antagonist. 1-Sar-8-Ile-Angiotensin 11 , on moderately high blood pressure under pentobarbital anesthesia. Vertical lines are standard errors of the means. ( $\boldsymbol{\square}$ ) represents the mean systemic blood pressure obtained before anesthesia. ( ${ }^{*}$ ) represents significant difference from the premedication-levels. 
produced. Additional $40 \% \mathrm{~g} / \mathrm{kg}$ i.v. injection of the agent 12 min after the first injection did not induce any significant changes in diastolic pressure, while a slight increase was observed in systolic pressure.

\section{Discussion}

Sodium pentobarbital has been found in the present experiments to increase heart rate in all cases regardless of changes in plasma catecholamines. The increase was not induced by a less depth of anesthesia simply because corneal reflexs were absent. Although the direct effects of $P B$ on the cardiac conduction system are depressive $(8,9)$, the presence of the normal sympathetic tone definitely increases the resistance of the conduction system to the depressant effects (8). It has also been reported that barbiturates attenuate reflexly induced parasympathetic stimulation (15). Anesthetic concentrations of PB have a greater inhibitory effect on the stimulated release of $A C h$ than on the $N E$ release in chicken heart (4). An increase in heart rate mediated by a decrease in vagal tone has been observed during mild exercise (16). Heart rate of conscious dogs is under profound vagal influence, and administration of methylatropine causes a sustained tachycardia (17). It is conceivable, therefore, that under PB anesthesia heart rate is essentially under sympathetic influence, and tachycardia due to a relative enhancement of sympathetic tone results in fact from withdrawal of parasympathetic activity. Nevertheless, since the increase in heart rate was depressed only slightly by propranolol, some explanation additional to the $\beta$-receptor stimulation must also be sought. The heart rate after anesthesia with propranolol in the present study is similar to that after autonomic nervous blockade in conscious human subjects (18) and may represent the high intrinsic heart rate.

Changes in the reuptake, nonspecific binding and catabolism of released $N E$ cannot be ruled out. However. PB does not inhibit the NE uptake (3), and plasma NE in the present experiments did not vary concomitantly with plasma Epi. Thus, the plasma NE levels obtained after anesthesia probably reflect neurosympathetic activities. A marked decrease in blood pressure just after $\mathrm{PB}$ injection may be induced by the depressant effects on NE release from the sympathetic nerves (3) and also on vascular smooth muscle (7). Following the transient drop, the blood pressure level increases to one of three distinct levels, probably depending on the concentration of plasma $P B$ and various reflex systems. The low (Group I) and the very high blood pressure levels (Group III) after PB anesthesia appear in part to reflect low and high neurosympathetic activities. respectively. On the other hand, plasma NE decreased in many cases where blood pressure increased moderately after anesthesia. One possibility for the cause of the moderately high blood pressure is an increase of the sympathetic adrenergic tone in the peripheral artery despite no detection of elevation of the plasma NE as suggested in other previous reports $(13,19)$. Because, in some cases where plasma NE levels in consciousness were relatively low. the level increased under anesthesia and 117-182 $\mathrm{pg} / \mathrm{ml}$ of plasma NE, probably definite basal levels under PB anesthesia, are not so low values. A relative enhancement of neurosympathetic tone which accompanies suppression of parasympathetic activity (4) may be partly involved. Pressor responses to exogenous NE were not influenced by propranolol-pretreatment, and the $\beta$ adrenoceptor action of NE was probably negligible under anesthesia. Another possibility thus manifests itself: a marked decrease in circulating Epi as a $\beta$-agonist may partly contribute to the moderately elevated levels of blood pressure. The increased level of 
plasma renin activity was maintained for at least $2 \mathrm{hr}$ after anesthesia, whereupon the third possibility emerges: angiotensin's role. However, the increases in plasma renin activity were observed regardless of the levels of blood pressure 30-60 min after anesthesia, and angiotensin $1 \mathrm{I}$ antagonist did not induce any decrease in moderately high blood pressure. The renin-angiotensin system appears not to be essential for the maintenance of a moderately high blood pressure during PB anesthesia, which coincides with Leenen and Provoost (13).

Plasma Epi showed an extreme drop after $\mathrm{PB}$ anesthesia and maintained the levels for at least $2 \mathrm{hr}$ in all cases. This result confirms earlier findings that an anesthetic concentration of PB strongly inhibits the catecholamine release from the chromaffin cells of the adrenal medulla (2, 5). These findings make it less likely that adrenal-medullary activity contributes to the maintenance of blood pressure after anesthesia. A less marked decrease in plasma Epi in the very high blood pressure group may represent a less inhibitory effect by $\mathrm{PB}$ on sympathoadrenal activity.

Changes in plasma DA induced by PB anesthesia are related to those in plasma NE rather than to those in plasma Epi. Its origin seems at least in part to be the peripheral sympathetic neuroeffector junction.

The current results also tell us that it is difficult to compare the pressor effect of exogenous NE as the degree of increase because the responsiveness to exogenous NE is dependent on the blood pressure levels.

In the present study, the increases in plasma renin activity following induction of anesthesia by $\mathrm{PB}$ was not accompanied with increases in plasma catecholamines. This result is consistent with the fact that the response of plasma renin activity to $P B$ is not mediated through the peripheral sympa- thetic nervous system via $\beta$-adrenoceptors. which we know because propranolol and sympathectomy do not prevent the response (11. 12). A fall in renal perfusion pressure produced by the initial decrease in blood pressure may partly cause the increase in plasma renin activity after PB anesthesia as suggested by Yun et al. (12).

Acknowledgements: We would like to thank Dr. E. Tsutsumi for his helpful advice about the catecholamine assay. We are grateful to Mr. I. Ichiyama for technical assistance and to Daiichi Seiyaku Co. for providing 1 -sarcosine, 8 -isoleucine angiotensin 11.

\section{References}

1) Vatner, S.F. and Braunwald, E.: Cardiovascular control mechanisms in the conscious state. $N$. Engl. J. Med. 293, 970-976 (1975)

2) Holmes, J.C. and Schneider, F.H.: Pentobarbitone inhibition of catecholamine secretion. Br. J. Pharmacol. 49, 205-213 (1973)

3) Göthert, M. and Rieckesmann, J.M.: Inhibition of noradrenaline release from sympathetic nerves by pentobarbital. Experientia 34, 382384 (1978)

4) Lindmar, R., Löffelholz, K. and Weide, W.: Inhibition by pentobarbital of the acetylcholine release from the postganglionic parasympathetic neuron of the heart. J. Pharmacol. Exp. Ther. 210, 166-173 (1979)

5) Walker, W.F., Zileli, M.S., Reutter, F.W., Shoemaker, W.C. and Moore, F.D.: Factors influencing the 'resting' secretion of the adrenal medulia. Am. J. Physiol. 197, 765-772 (1959)

6) Picotti, G.B., Carruba, M.O., Galva, M.D., Ravazzani, C., Bondiolotti, G.P. and DaPrada, M.: Drug-induced changes of plasma catecholamine concentrations. In Radioimmunoassay of Drugs and Hormones in Cardiovascular Medicine, Edited by Albertini, A., DaPrada, M. and Peskar. B.A.. p. 133-148. Elsevier/North Holland Biomedical Press. Amsterdam (1979)

7) Altura, B.T. and Altura, B.M.: Pentobarbital and contraction of vascular smooth muscle. Am. J. Physiol. 229, 1635-1640 (1975)

8) Urthaler, F., Krames, B.L. and James, T.N.: Selective effects of pentobarbital on automaticity and conduction in the intact canine 
heart. Cardiovasc. Res. 8, 46-57 (1974)

9) Chiba, S.: Different chronotropic and inotropic effects of pentobarbital in the blood-perfused. isolated dog atrium. Eur. J. Pharmacol. 38, 89-94 (1976)

10) DeChamplain, J., Farley, L., Cousineau, D. and Ameringen, M.R.V.: Circulating catecholamine levels in human and experimental hypertension. Circ. Res. 38, 109-114 (1976)

11) Ganong, W.F.: Sympathetic effects on renin secretion: Mechanism and physiological role. Adv. Exp. Med. Biol. 17, 17-32 (1972)

12) Yun, J.C., Kelly, G.D. Bartter, F.C. and Tade, J.E.: Mechanism for the increase in plasma renin activity by pentobarbital anesthesia. Life Sci. 22, 1545-1554 (1978)

13) Leenen, F.H.H. and Provoost, A.P.: Maintenance of blood pressure by $\beta$-adrenoceptor mediated renin release during different forms of anesthesia in rats. Can. J. Physiol. Pharmacol. 59, 364-370 (1981)

14) Kawasaki, H., Cline, W.H., Jr. and Su, C.: Enhanced angiotensin-mediated facilitation of adrenergic neurotransmission in spontaneously hypertensive rats. J. Pharmacol. Exp. Ther. 221, 112-116 (1982)

15) Higgins, C.B., Vatner, S.F. and Braunwald, E.: Parasympathetic control of the heart. Pharmacol. Rev. 25, 119-155 (1973)

16) Christensen, N.J. and Brandsborg, O.: The relationship between plasma catecholamine concentration and pulse rate during exercise and standing. Eur. J. Clin. Invest. 3, 299-306 (1973)

17) Lokhandwala, M.F., Cavero, I., Buckley, J.P. and Jandhyala, B.S.: Influence of pentobarbital anesthesia on the effects of certain autonomic blocking agents on heart rate. Eur. J. Pharmacol. 24, 274-277 (1973)

18) Jose, A.D. and Taylor, R.R.: Autonomic blockade by propranolol and atropine to study intrinsic myocardial function in man. J. Clin. Invest. 48, 2019-2031 (1969)

19) Vlachakis, N.D., Alexander, N. and Maronde, R.F.: Increased plasma normetanephrine in spontaneously hypertensive rats. Clin. Exp. Hypertens. 2, 309-319 (1980) 